\title{
La Drépanocytose Chez Les Enfants Hospitaliers Au Service De Pédiatrie (CHR El Idriss De Kénitra, Maroc): A Propos De 53 Cas.
}

\section{Belala A. \\ Marc I.}

Laboratoire de Biotechnologie et Environnement, Département de Biologie, Faculté des Sciences, Université Ibn Tofail, Kénitra, Maroc

\section{Hajji. A.}

Service de pédiatrie centre hospitalier régional El Idrissi de Kenitra

\section{Belghyti. D. \\ El kharrim. $K$.}

Laboratoire de Biotechnologie et Environnement, Département de Biologie,

Faculté des Sciences, Université Ibn Tofail, Kénitra, Maroc

Service de pédiatrie centre hospitalier régional El Idrissi de Kenitra

\section{doi: 10.19044/esj.2016.v12n12p201 URL:http://dx.doi.org/10.19044/esj.2016.v12n12p201}

\begin{abstract}
The objective of this work is to study sickle cell in children 1 to 16 years at El Idrissi regional hospital of Kenitra (Morocco) The study was conducted between March and June 2012. The population includes 53 patients, 39 male and 14 female. The studied parameters were age, sex, socioeconomic status, origin, age of diagnosis and schooling. Data on sickle cell patients was collected from information sheets available from the archives department at the El Idrissi regional hospital of Kenitra and a patient's verbal consent or that of the child's parents. Sickle cell disease is the third leading cause of admission (10.16 \% of total admissions). $47.16 \%$ of patients are under the age of 6 years. The diagnosis of disease is made in $32.07 \%$ of the cases around 9 months. In $94.3 \%$ of cases, patients are from family whose father has a low and irregular income. The main causes of hospitalization are painful crises (69.81\%), acute anemia (28.30\%), and infections (28.30\%). As a medical treatment, the rehydration, analgesics, antibiotics and blood transfusions are the most prescribed. The transfusion was performed in $96.22 \%$ of $\%$ cases. The lack of early detection and socio -economic pressures and environmental conditions seems to put our patients in a vulnerable state.The focus should be on prevention and strengthening the capacity of families.
\end{abstract}


Keywords: Sickle cell disease, child, hospital, epidemiology, Kénitra

\section{Résumé}

L'objectif de ce travail est d'étudier la drépanocytaire chez les enfants de 1 à 16 ans au centre hospitalier régional El Idrissi de Kénitra (Maroc). L'étude a été menée entre Mars et Juin 2012. La population comporte 53 patients, 39 de sexe masculin et 14 de sexe féminin. Les paramètres étudiés sont : l'âge, le sexe, le niveau socio-économique, l'origine, l’âge de diagnostic, scolarité. Les données concernant les patients drépanocytaires ont été recueillis à partir des fiches de renseignements disponibles auprès du service des archives au centre hospitalier régional El Idrissi de Kénitra et un consentement verbal du patient ou de celui des parents de l'enfant. La drépanocytose est la troisième cause d'admission (10,16\% des admissions totales). 47,16 \% des sujets sont âgés de moins de 6 ans. Le diagnostic de la maladie est fait dans 32,07\% des cas autour de 9 mois. Dans 94,3 \% des cas, les patients sont issus de famille dont le père a un revenu faible et irrégulier. Les principales causes d'hospitalisation sont les crises douloureuses $(69,81 \%)$, les anémies aigues $(28,30 \%)$, et les infections (28,30\%). La réhydratation, les antalgiques, les antibiotiques et les transfusions sanguines sont les plus prescrits comme traitement médical. La transfusion a été pratiquée dans 96,22 \% des cas. L’absence de dépistage précoce et les pressions socio-économique et environnementale semble être la vulnérabilité de nos patients. L'accent doit être mis sur la prévention et le renforcement des capacités des familles.

Mots clés : Drépanocytose, enfant, milieu hospitalier, épidémiologie, Kénitra.

\section{Introduction}

La drépanocytose est la maladie génétique la plus répandue dans le monde (WHO, 2008). Elle résulte d'une mutation dans le gène de la $\beta$ globine qui provoque la synthèse d'une hémoglobine anormale, l'hémoglobine S (Hb S). En situation d'hypoxie, la polymérisation de cette $\mathrm{Hb} \mathrm{S}$ est à l'origine de phénomènes vaso-occlusifs et d'une anémie hémolytique chronique pouvant entraîner un infarcissement progressif de la rate.

La drépanocytose est particulièrement fréquente chez les personnes originaires d'Afrique subsaharienne, d'Inde et de pays méditerranéens. Néanmoins, du fait des mouvements de migrations récents, cette hémoglobinopathie est présente dans le monde entier. Près de 120 millions de personnes seraient porteuses d'une mutation drépanocytaire (Bardarkdjian 
et al., 2004) et environ 50 millions de personnes seraient touchées par cette maladie (Baene 2008).

L’Organisation Mondiale de Santé (OMS) évalue à 275000 le nombre d'enfants qui naissent chaque année atteints de drépanocytose (Modell et al., 2008). La drépanocytose, malgré son ampleur, reste une maladie très peu connue. Ce n'est que le 21 décembre 2008 qu'elle a été reconnue comme quatrième priorité de sante' publique par l'Assemblée générale des Nations Unies (WHO, 2008).

C'est une pathologie répandue en Afrique où elle pose d'importants problèmes sanitaires. Son intrication avec les pathologies infectieuses et parasitaires lui confère une gravité particulière, en contribuant à un taux de mortalité infantile élevée (http://medecinetropicale.free.fr/cours/hemoglobinoses.pdf).

$\mathrm{Au}$ Maroc, il n'existe pratiquement pas, pour des raisons économiques, de système de prise en charge diagnostique et thérapeutique orienté vers le long terme. C'est ainsi que la plupart des enfants atteints de cette maladie n'arrivent pas à l'âge adulte. Il nous a donc paru intéressant de relever par l'étude de dossiers médicaux, les particularités cliniques et thérapeutiques actuelles de la maladie, ainsi que le bénéfice à escompter d'une prise en charge suivie, lorsqu'elle est accessible.

En nous aidant des données actualisées de la littérature, nous avons souhaité approfondir le problème de la maladie drépanocytaire dans des domaines aussi variés que ceux de la génétique, de l'épidémiologie et des perspectives thérapeutiques. Enfin, à travers ce travail, nous aurons cherché à élucider une pathologie mondialement rencontrée, riche en enseignement lourde de conséquences.

\section{Milieu d'étude}

Afin d'étudier les aspects cliniques, biologiques et socioéconomiques de la drépanocytose en pédiatrie à l’hôpital El IDRISSI de Kénitra, nous avons analysé les dossiers de 53 patients porteurs de syndromes drépanocytaires majeurs et dont le diagnostic a été confirmé par une électrophorèse l'Hb, suivis à l'hôpital El IDRISSI de Kénitra de Mars au Juin 2012.Un consentement verbal des parents ou tuteurs était obligatoirement sollicité.

Dans le cadre d'atteindre les objectifs de cette étude, on a élaboré un questionnaire qui aborde les thèmes suivants :

- Arguments épidémiologiques,

- Arguments cliniques,

- Arguments biologiques,

- Prise en charge. 
Tous les renseignements utiles à notre étude ont été recueillis dans des tableaux ou « fiches d'exploitation ».

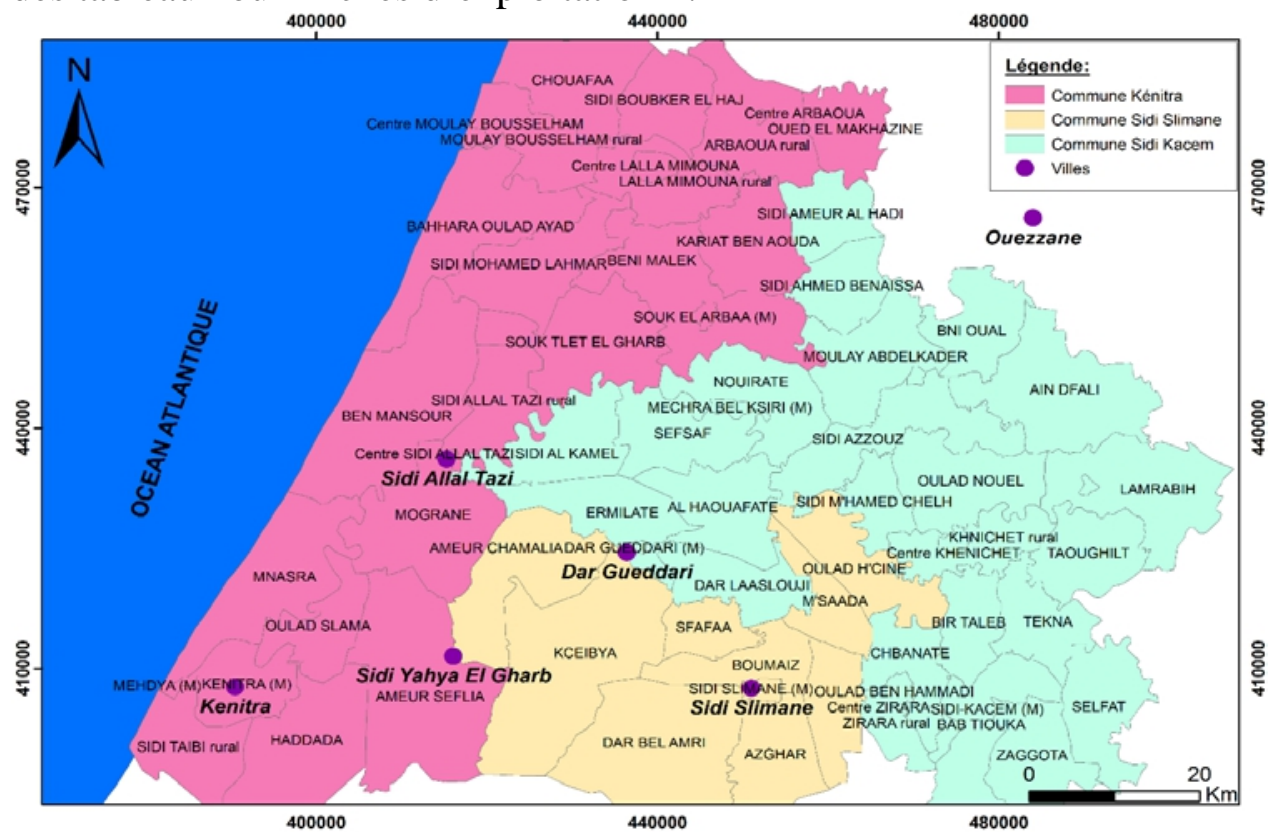

Figure 1 : Découpage administratif de la région du Gharb-Cherarda- Beni Hssen

(Direction de l'aménagement du territoire, 2008)

\section{Population}

Tableau 1 : Données descriptif de l'échantillon.

\begin{tabular}{|c|c|c|c|}
\hline & & & Total \\
\hline Age & $<10$ ans $: 39$ & $>10$ ans $: 14$ & 53 \\
\hline Sexe & Masculin $: 39$ & Féminin $: 14$ & 53 \\
\hline Milieu & Urbain $: 10$ & Rural $: 43$ & 53 \\
\hline Motifs d'hospitalisation & crises douloureuses & 31 & 53 \\
\cline { 2 - 3 } & les infections & 15 & \multirow{2}{*}{} \\
\cline { 2 - 3 } & les anémies aigues & 7 & \\
\hline
\end{tabular}

\section{Méthodes d'analyse statistique}

Pour l'analyse statistique, les données ont été saisies et analysées par le logiciel SPSS (Version 10). Les résultats obtenus ont été rapportés dans des tableaux comparatifs, des courbes et des diagrammes. Leur analyse a été faite selon les techniques épidémiologiques usuelles Taux, médiane, écart type,....

\section{Résultats}

La drépanocytose est la troisième cause d'admission à l'hôpital El Idrissi. On constate que l'âge de l'échantillon est de 7,1 $\pm 3,7$ avec des extrêmes de 1 et 16 ans (Tableau 2). 
L'échantillon est constitué de 26,41 \% de filles ( $\mathrm{N}=14)$ et de 73, 58 $\%$ de garçons $(\mathrm{N}=39)$.

Tableau 2 : Répartition des patients en fonction de l'âge.

\begin{tabular}{|c|c|c|c|c|}
\hline & Moyen d’âge & écart type & $\max$ & $\min$ \\
\hline Total (53) & 7,1 & $\pm 3,7$ & 16 & 1 \\
\hline
\end{tabular}

Les enfants ayant un âge inférieur ou égal à 3 ans représentent 16,98 \% des enfants enquêtés. Les autres tranches d'âge sont respectivement de l'ordre de $30,18 \% ; 26,41 \% ; 20,93 \%$; 9,43\% pour les tranches d'âge de 3 à 6 ans, de 6 à 9 ans, de 9 à 12 ans et de 12 à 16 ans.

Tableau 3 : Répartition des patients en fonction des classes d'âge.

\begin{tabular}{|c|c|c|}
\hline Tranches d'âge & Effectifs des malades & Pourcentage \% \\
\hline De 0 à 3 ans & 9 & 16,98 \\
\hline De 3 à 6 ans & 16 & 30,18 \\
\hline à 9 ans & 14 & 26,41 \\
\hline De 9 à 12 ans & 9 & 20,93 \\
\hline De 12 à 16 ans & 5 & 9,43 \\
\hline
\end{tabular}

Les patients provenaient dans la majorité des cas des communes Mnasra, Kénitra et Ben Mansour avec respectivement 33,96\%, 18, 86\% et $15,09 \%$.

Tableau 4 : Répartition des patients selon la provenance.

\begin{tabular}{|lcr|}
\hline Provenance & Effectifs des malades & Pourcentage\% \\
\hline Mnasra & 18 & 33,96 \\
\hline Ben Mansour & 8 & 15,09 \\
\hline Moulay Bouslham & 5 & 9,43 \\
\hline Sidi Yahia & 6 & 11,32 \\
\hline Sidi Slimane & 4 & 7,54 \\
\hline Allal Tazi & 2 & 3,77 \\
\hline Kénitra & 10 & 18,86 \\
\hline Total & 53 & 100,00 \\
\hline
\end{tabular}

Le pourcentage des enfants enquêtés d'origine rurale 81,13\% (43/53) est plus élevé par rapport à celui calculé chez les enfants d'origine urbaine18, 86\% (10/53) (figure 2). 


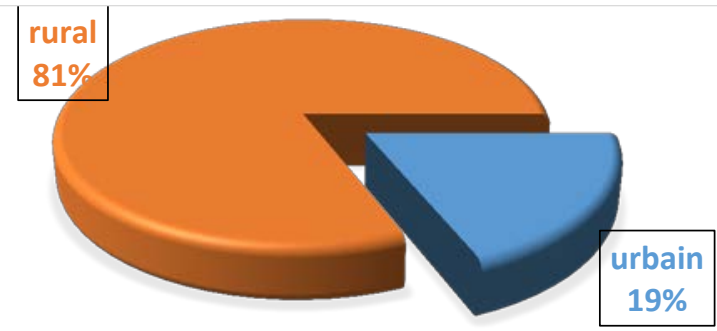

Figure 2 : Répartition des drépanocytaires selon le milieu de résidence chez les enfants hospitalier dans l’hôpital El Idrissi de Kénitra (Maroc) en 2012

La majorité des familles des enfants enquêtés ont un revenu familial irrégulier 94,33\% (50/53), alors que seulement 5,66 \% (3/53) ont un revenu familial régulier.

La consanguinité n’a été précisée que chez 24 des 53 cas étudiés, soit un pourcentage de $45,3 \%$ (figure 3 ).

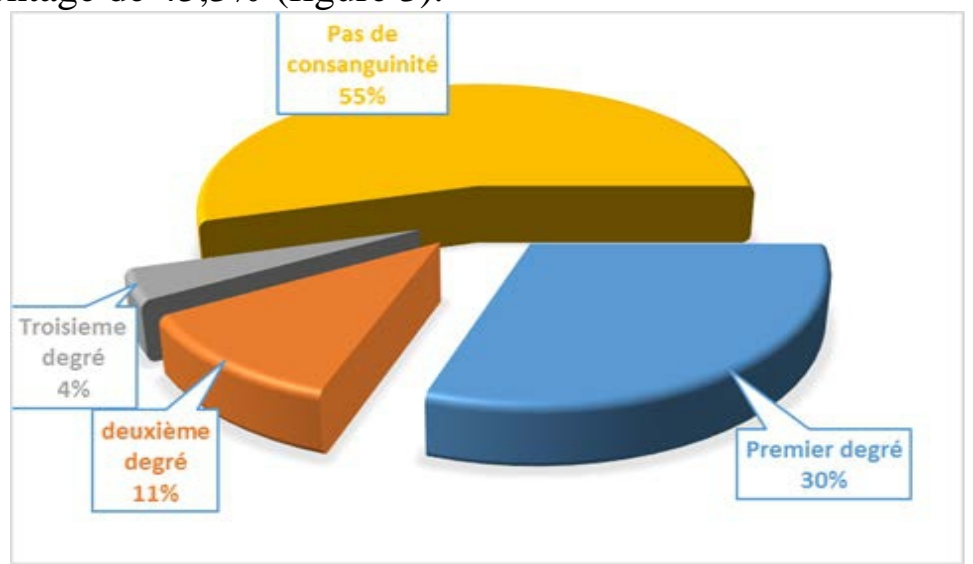

Figure 3 : Répartition des malades enquêtés selon la consanguinité chez les enfants hospitaliers dans l’hôpital El Idrissi de Kénitra (Maroc) en 2012.

L'âge de diagnostic se situe entre quelques mois à 12 ans avec un pic à 1 an et une médiane d'âge de 1 an (figure 4). 


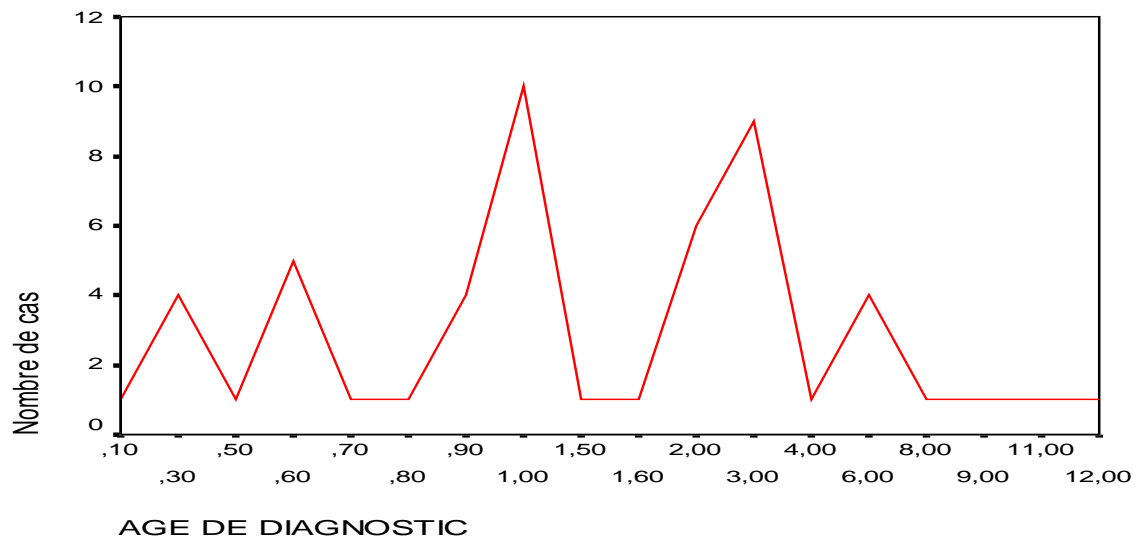

Figure 4 : Répartitions drépanocytaires selon l’âge de diagnostic chez les enfants hospitaliers dans l’hôpital El Idrissi de Kénitra (Maroc) en 2012.

D'après l'analyse statistique qui a était faite par le test student, on constate que l’âge de diagnostic n'est pas lié significativement au sexe des drépanocytaires $\mathrm{p}<0,05$. Aucune différence significative n’a été notée entre l'âge de diagnostic et la consanguinité.

Tableau 5 : Résultats par analyse statistique des liens entre le sexe et l'âge de diagnostic.

\begin{tabular}{|c|c|c|c|c|}
\hline & La Moyenne & \pm Ecart-type & Seuil de signification & Décision \\
\cline { 1 - 3 } $\begin{array}{c}\text { Masculin } \\
\text { (N=39) }\end{array}$ & 2,57 & $\pm 2,92$ & $\begin{array}{c}\mathrm{P}=0,17 \\
(<0,05)\end{array}$ & (NS) \\
\cline { 1 - 2 } $\begin{array}{c}\text { Féminin } \\
(\mathbf{N}=14)\end{array}$ & 2,11 & $\pm 1,96$ & & \\
\hline
\end{tabular}

Tableau 6 : Résultats par analyse statistique des liens entre la consanguinité et l’âge de diagnostic.

\begin{tabular}{|c|r|r|c|c|}
\hline & La Moyenne & \pm Ecart-type & $\begin{array}{c}\text { Seuil de } \\
\text { signification }\end{array}$ & Décision \\
\hline Consanguinité & 2,83 & $\pm 1,37$ & $\begin{array}{c}\mathrm{P}=0,75 \\
(<0,05)\end{array}$ & (NS) \\
\hline Age de diagnostic & 2,45 & $\pm 1,69$ & \\
\hline
\end{tabular}

$94,33 \%$ des patients ont des frères et des sœurs. Parmi ces frères et sœurs $26,50 \%$ sont drépanocytaires et 10,84\% sont décédés à cause de la maladie.

Le groupe sanguin a été précisé chez les malades en se référant au centre régional de transfusion de Kénitra. 39,6 \% ont un groupe sanguin A ; $30,2 \%$ du groupe $\mathrm{O} ; 24,5 \%$ du groupe $\mathrm{B}$ et $5,7 \%$ du groupe $\mathrm{AB}$.

Compte tenu des différences d'âges parmi les enfants enquêtés, les niveaux scolaires sont différents, ils se répartissent de la façon suivante : presque 47,16\% de la population étudiée étaient des enfants au foyer qui non pas encore atteint l'âge de la scolarisation ; 9,43\% sont non scolarisés à 
cause de la maladie ; 39,62\% ont un niveau scolaire primaire, par ailleurs $3,77 \%$ sont des collégiens (figure 5).

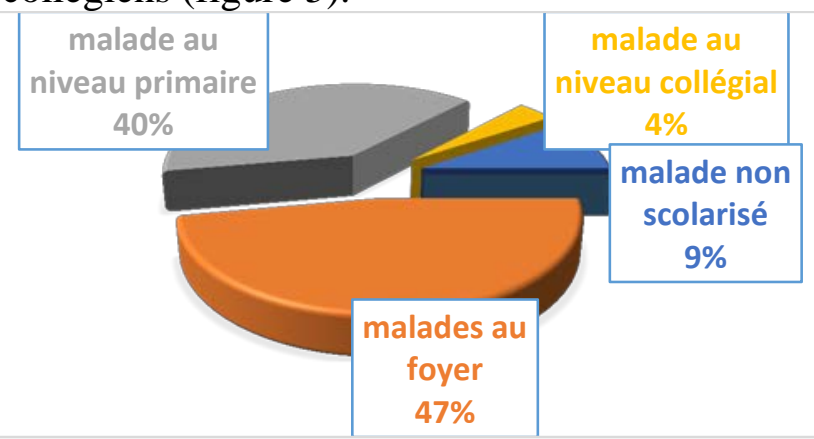

Figure 5 : Répartition des drépanocytaire selon le niveau scolaire.

92,45\% des malades scolarisés ont déclaré que leur scolarité est perturbée par la maladie et qu’ils ont été obligés de redoubler au moins une année.

\section{Discussion et conclusion}

La drépanocytose est la troisième cause d'admission à l'hôpital (10,16\% des admissions totales) ce qui rejoint les observations faite par Thuilliez (1997). Cette population observée est constituée de 26,4\% de filles et $73,6 \%$ de garçons. On observe une prédominance masculine avec un sexratio de 2,78, d'autres auteurs dont Diagne et al.,(2000) au Sénégal et Mabiala et al.,(2005) rapportent une légère prédominance du sexe masculin, par contre, d'autres ne constatent aucune prédominance entre les deux sexes ; c'est le cas de Thuilliez et al.,(1997) et Dreux(2012), en revanche, Nacoulma et al.,(2006) au Burkina Faso ont rapporté une prédominance féminine avec un sex-ratio de 0,90. Les cas rapportés par les différents auteurs seraient en rapport avec les données démographiques de chaque pays car génétiquement la transmission de l'HbS n'est pas liée au sexe.

Le diagnostic de la maladie est fait dans 20,75 \% des cas autour de 6 mois. Ce constat traduit un retard au dépistage et donc à la prise en charge. En effet, les hospitalisations pour crises vaso-occlusives et les complications infectieuses ont constitué, dans la grande majorité des cas, les circonstances de découverte de la maladie. Le dépistage néonatal systématique permettrait une prise en charge précoce et une amélioration du pronostic vital des drépanocytaires.

$81,13 \%$ des sujets sont issus des milieux ruraux et se répartissaient de façon inégale, avec des localités très touchées (Ben Mansour et Mnasra) qui pourrait être due à la fréquence de la consanguinité dans cette région, dont le taux atteint 45,3\%. Ce qui constitue un facteur favorisant l'éclosion des formes homozygotes et doubles hétérozygotes de la maladie ceci rejoint des 
observations faites en Tunisie Mseddi et al., (1999), et justifient l'élaboration d'une stratégie de lutte qui devrait être axée essentiellement sur les moyens prophylactiques.

Concernant le statut socioprofessionnel des parents, 94,33\% des cas les patients sont issus de famille dont le père a un revenu faible et irrégulier. Le statut socioprofessionnel du père est habituellement utilisé pour apprécier le niveau de vie des familles bien qu'il ne le traduise pas en réalité. Dans notre étude, comme chez Diagne et al.,(2000) au Sénégal la proportion élevée des pères à revenus irréguliers et les revenus relativement faibles chez les salariés permettent de mesurer le poids du bas revenu familial sur la qualité de la prise en charge. Cependant, les sacrifices fournis par les parents permettent une régularité relative du suivi de nos patients. Si les patients arrivaient à faire toutes les vaccinations recommandées, certains n'étaient revus que lors des crises.

Le fait que cette maladie soit inconnue, hors du milieu des malades et du milieu médical rend la vie des jeunes patients souvent difficile et particulièrement dans le monde scolaire d'autant que les actes de gestion de la maladie ont un grand caractère de visibilité Nous avons cherché à savoir si cette maladie avait perturbé la scolarité de ces enfants, il en ressort que 92,45 $\%$ de ces enfants ont eu une scolarité perturbée contre 7,55\% qui ne semblent avoir surmontes leur maladie. Près de $78,26 \%$ déclarent avoir eu une scolarité perturbée, ont été amenés à redoubler au moins une fois une classe. Plus de 9,43\% des enfants n’ont jamais été scolarisés à cause de la maladie.

A terme de ce travail, il se dégage que la drépanocytose est un problème réel de santé publique. La maladie est très fréquente dans les populations d'origine rurale, la répartition des patients selon l'âge fait apparaitre que les garçons sont plus touchés que les filles, seulement 32,07\% de ces enfants ont été diagnostiqué avant 9 mois.

Chez nos patients, les crises douloureuses intenses, les infections, les épisodes d'anémie aiguë sont les complications aiguës les plus fréquentes. Le traitement conventionnel est essentiel par : antibiothérapie et vaccinations, antalgiques, transfusion sanguine. Les enfants drépanocytaires au cours de leur scolarité, et à cause des crises douloureuses sont régulièrement contraints d'interrompre leur cursus. Il s'accompagne d'échecs et plus tard d'abandons scolaires.

Nos résultats de suivi méritent cependant d'être renforcés par la mise en œuvre, à court terme, au niveau du centre hospitalier El Idrissi de Kénitra, d'actions telles que la création d'une unité de vaccination, la réalisation du dépistage néonatal et la systématisation de l'antibioprophylaxie. À moyen terme, il est nécessaire de mettre en place un programme national de prise en charge de la drépanocytose afin de participer à l'atteinte de l'objectif fixé par 
l'OILD (Organisation Internationale de Lutte contre la Drépanocytose) de réduire de $40 \%$ la mortalité des enfants drépanocytaires de moins de 5 ans.

\section{References:}

http://medecinetropicale.free.fr/cours/hemoglobinoses.pdf

Modell B., Darlison M., 2008. Global epidemiology of haemoglobin disorders and derived service indicators. Bulletin of the World Health Organization (BLT) 86(6):480-487.

United Nations. Sickle cell anemia. In: 63rd General assembly plenary; 2008, http://www.un.org/News/Press/docs/2008/ga10803.doc.htm

Nacoulma EWC., Sakande J., Kafando E., Kpowbié ED.\& Guissou IP. 2006. Profil hématologique et biochimique des drépanocytaires SS et SC en phase stationnaire au Centre Hospitalier National Yalgado Ouedraogo de Ouagadougou. Mali Medical, 21(1):8-11.

Diagne I., Ndiaye O., Moreira C., Stignate-Sy H., Camara B. \& Diouf S.2007. Les syndromes drépanocytaires majeurs en Pédiatrie à Dakar (Sénégal) Arch Ped, 7:16-24.

Mabiala BJR., Nkanza KSAT., Ganza ZPS., Nzingoula S. \& Senga P.2005 .Causes d'hospitalisation des enfants drépanocytaires: influence de l'âge (CHU de Brazzaville, Congo) Bull Soc Pathol Exot ,98(5) :392-93.

Thuilliez V., Vierin Y.1997. Le poids de la drépanocytose en milieu pédiatrique au Gabon.Santé publique. 1(9) :45-60.

Dreux O. Grenoble (France) : Université Joseph Fournier ; 2012. Education thérapeutique pour les enfants drépanocytaires : justifications à la mise en place et initiation de ce projet au CHU de Grenoble. Thèse de médecine.

Nacoulma EWC., Bonkoungou P., Dembelele Ye D. \& Kam L.2006.Les drépanocytoses majeures dans le service de pédiatrie du centre hospitalier universitaire Sounou Sanon de Bobo-Dioulasso. Med Afr Noire, 53(12) :694-98.

Bardarkdjian J., Wajcman H.2004. Epidémiologie de la drépanocytose. La revue du praticien (54):1531-1533.

Baene L. La drépanocytose. [en ligne]. Disponible sur : http://www.caducee.net/Dossierspecialises/genetique/drepanocytose.asp (29/09/2008).

Tournebize S., RABIER R.1997. Vivre avec la drépanocytose Antiane, $\mathrm{n}^{\circ}$ 35, sept 97 ; 13-16, INSEE éditeur.

Nacoulma EWC., Kam L. \& Gué EE. 2006. Évaluation du statut vaccinal de l'enfant drépanocytaire de la ville d’Ouagadougou (Burkina Faso). Santé, 16: 155-60.

Mseddi S., Gargouri J.\& Labiath Z.,1999. Prévalence des anomalies de l'hémoglobine à Kebili (Sud Tunisien). Revu Epidemio Santé Public, 47 :2936. 